\title{
Determining the Representative Volume Element Size for Three-Dimensional Microstructural Material Characterization. Part 2: Application to Experimental Data
}

\author{
William Harris and Wilson K. S. Chiu* \\ Department of Mechanical Engineering \\ University of Connecticut \\ 191 Auditorium Road \\ Storrs, CT 06269-3139 \\ * Corresponding Author, E-mail: wchiu@engr.uconn.edu.
}

\begin{abstract}
Improved microstructural imaging and characterization methods have recently opened the door for quantitative evaluation of microstructures of such functional materials as solid oxide fuel cell and battery electrodes and composite gas separation membranes. Accurate quantitative characterization of these structures relies on the concept of a representative volume element (RVE) to provide a sufficiently large sample to be statistically representative of the material. In Part 1 of this work, several models were described to determine the RVE size for several common microstructural properties: volume fraction, particle size, and network contiguity. In this work, extensive synchrotron $\mathrm{x}$-ray nanotomography imaging of a multiphase composite gas separation membrane is used to provide an experimental comparison to the model predictions. Results suggest that the models provide a reasonable estimate of RVE size, and can serve as a starting point for researchers planning imaging and characterization experiments.
\end{abstract}

\section{Introduction to Experimental RVEs}

Numerous experimental studies have aimed to quantify an RVE size for material characterization. Frequently, the evaluation of volume-independence is performed after imaging either by altering the analysis volume or splitting the total sample volume into numerous smaller

(C) 2015. This manuscript version is made available under the Elsevier user license http://www.elsevier.com/open-access/userlicense/1.0/ 
sub-volumes and analyzing the distribution of characterization results. Several such studies are briefly described here to provide context for the following discussion of the characterization performed in this work. Shearing et al. used x-ray tomography to examine the microstructure of a graphite electrode for a lithium-ion battery.[1] After imaging, the domain was sub-divided into a number of smaller sub-volumes of different sizes to analyze the variation in properties. An RVE was defined when the standard deviation of results at a given size was considered adequately small, although this criteria was not explicitly defined. In this manner the authors determined an RVE size for specific surface area to occur for a volume of 43×60x60 microns, and for tortuosity to be at least as large as the entire sample, $43 \times 348 \times 478$ microns. Although not stated in the paper, the mean particle size visually appears to be about 20-30 microns based on a 3-D rendering.

Metcalfe et al. used a simulation approach to generate and analyze structures of composite solid oxide fuel cell electrodes.[2] The group was most interested in the triple phase boundary length (TPBL), and defined an RVE as existing when less than 3\% variation existed amongst replicate volumes of the same ratio of pore, ionic, and electronic phases. Such a criteria was used to determine a cubic RVE volume of $L^{*}=14$, where $L^{*}$ is defined below in Eq. 1 . Measurements of the effective TPBL based on only contiguous structures were also performed at a single composition and provided similar results. The authors also acknowledged that significant variations of the effective TPBL could exist when one of the composite's phases is near its percolation threshold, increasing the necessary size of the RVE.

Lombardo et al. used x-ray nanotomography to evaluate a variety of properties for a composite SOFC anode composed of nickel and yttria-stabilized zirconia (YSZ).[3] The approach evaluated a single cube for several different values of $\mathrm{L}^{*}$, and defined an RVE when 
the characterization results did not vary significantly between successive $L^{*}$ sizes. Such an approach can suffer from a dependence on the step size between successive $\mathrm{L}^{*}$, especially if the step is made small, and does not rigorously evaluate replicate sub-volumes for each $\mathrm{L}^{*}$. Nonetheless, large YSZ particles were found to cause significant fluctuations in the results for low values of $\mathrm{L}^{*}$, and it was concluded that particle size distribution, tortuosity, and properties involving the YSZ phase required the largest RVE's, in excess of roughly L* 6.

Laurencin et al. also used x-ray tomography to study Ni-YSZ, but used a slightly different imaging procedure capable only of distinguishing solid from pore.[4] A large total volume was imaged, which was split into numerous smaller sub-volumes to analyze the variation in porosity and tortuosity for different sub-volume size. For both properties, an RVE was obtained for $L^{*}=14.6$ based on sufficiently small variation of results.

Joos et al. used FIB-SEM imaging to characterize an SOFC cathode consisting of a porous solid phase of uniform composition.[5] The imaged volume was split into 3 smaller subvolumes and characterized in terms of porosity, tortuosity, and specific surface area. The size of the 3 sub-volumes was varied and RVE size was determined based on the variations between the sub-volumes as well as the results for different sub-volume sizes. It was reported that the porosity and tortuosity of the pore phase could be measured using a volume of $5.25 \times 5.25 \times 10$ microns, while measuring tortuosity of the solid phase required 5.25x5.25x20 microns. An RVE for specific surface area was not conclusive as the results showed variation even at the largest sub-volume sizes. The solid particle size is reported to be about 0.5 microns.

Rolland du Roscoat et al. and Gelb et al. each used x-ray tomography to examine the microstructures of paper and shale, respectively.[6,7] Each of the authors sub-divided their entire imaged volume into sets of smaller volumes of a range of sizes. The variation of properties 
measured on replicate volumes of a common size, as well as a required level of precision, were used to determine an appropriate representative volume size for porosity (Rolland du Roscoat and Gelb) and specific surface area (Rolland du Roscoat). The materials examined by these authors are much different than the particle-like composites considered in this work, but are mentioned because the overall experimental approach is very similar to that which is employed in the following sections of this paper.

\section{X-ray Nanotomography Imaging}

Composite oxygen separation membranes were fabricated using the same starting materials and general fabrication process as described in previous work.[8] The membranes consist of an oxygen ion-conducting gadolinium-doped ceria $\left(\mathrm{Ce}_{0.8} \mathrm{Gd}_{0.2} \mathrm{O}_{2}\right.$, GDC) phase and an electron conducting cobalt iron oxide $\left(\mathrm{CoFe}_{2} \mathrm{O}_{4}, \mathrm{CFO}\right)$ phase. The previous work also revealed an additional solid phase, denoted GFCC (suspected formula $\mathrm{Gd}_{0.374} \mathrm{Ce}_{0.079} \mathrm{Co}_{0.077} \mathrm{Fe}_{0.47} \mathrm{O}_{\mathrm{x}}$ ), which emerges during high temperature sintering in the membrane fabrication process. The same sample preparation and imaging procedures were used in this work to examine membranes fabricated under 5 sets of processing conditions, four of which are listed below:

1) $50 / 50 \mathrm{GDC}-\mathrm{CFO}$ ratio, sintered at $1300^{\circ} \mathrm{C}$ for 2 hours

2) $60 / 40 \mathrm{GDC}-\mathrm{CFO}$ ratio, sintered at $1300^{\circ} \mathrm{C}$ for 2 hours

3) $80 / 20 \mathrm{GDC}-\mathrm{CFO}$ ratio, sintered at $1300^{\circ} \mathrm{C}$ for 2 hours

4) $80 / 20 \mathrm{GDC}-\mathrm{CFO}$ ratio, sintered at $1300^{\circ} \mathrm{C}$ for 5 hours

The fifth set of conditions (80/20 GDC-CFO ratio, sintered at $1400^{\circ} \mathrm{C}$ for 2 hours) produced samples with very large feature sizes compared to the other four conditions. Presumably this is due to the higher sintering temperature, and is an area of ongoing 
investigation. Because of the large features, nanotomography measurements could only be performed on samples containing a small number of microstructural features. This comparison of length scales can be cast in terms of a dimensionless cubic sample edge length

$$
L^{*}=\frac{\text { CubeEdgeLength }[\mu m]}{\text { ParticleSi ze }[\mu \mathrm{m}]}
$$

In Eq. 1, the size of a cubic sample is scaled by some characteristic particle size of the material, as chosen by the researcher. The concept of a "particle" is used as an abstraction in this work to represent feature sizes in the material, and will be discussed further in application to this particular experimental work in section 3 . In this light, the membranes produced under the fifth set of processing conditions correspond roughly to samples of size $\mathrm{L}^{*}=1$ or 2 . Therefore, the samples are certainly too small to provide representative volumes, and are omitted from this study.

Three cylindrical samples of each of the other four processing condition were prepared by FIB-SEM milling followed by x-ray nanotomography imaging performed at the Stanford Synchrotron Radiation Lightsource (SSRL, beamline 6-2c) and the National Synchrotron Light Source (NSLS, beamline X8C).[3] The size of the prepared cylinders was approximately 10 microns in diameter and 15 microns in height. This size was chosen so that the sample would both provide partial transmission of the $\mathrm{x}$-ray beam as well as remain within the microscope field of view, however does not guarantee that volume independence will be achieved with that size. As in the previous work, nanotomography was performed spanning the Fe k-edge and ${\mathrm{Gd} \mathrm{L}_{3}-}^{-}$ edge to map GDC, CFO, GFCC, and pore phases. At both beamlines, a zone plate lens providing $30 \mathrm{~nm}$ spatial resolution was used. Microscope configuration and imaging parameters were similar for each sample, but slight adjustments were made according to the capabilities of the particular beamline. For example, when experimental time was constrained, imaging was 
performed at the 3 energy levels used previously: 7090, 7200, and $7280 \mathrm{eV}$. But when time was not a factor, such as due to the shorter exposure times required per image at SSRL, tomography could be performed at 4 energy levels to more precisely span each of the absorption edges: 7095 , 7130, 7230, and $7260 \mathrm{eV}$.

The image processing for each sample was performed in the same fashion as the previous work. The data sets were reconstructed using a filtered back-projection algorithm, and contrast across the absorption edges was used to generate seeds for a watershed segmentation algorithm implemented in MATLAB. The output of the watershed is 3-D digitized structures with the phases denoted by an integer label of 1, 2, 3, or 4 on a Cartesian grid.

\section{Statistical Characterization Approach}

Testing the existence of an experimental RVE requires sampling random and independent cubic sub-volumes of the material and observing the variation in properties among them. Because each cylindrical GDC-CFO sample was extracted from a random location on the membrane, each selected sub-volume from within the cylinder can also be considered random. But particular attention must be paid to ensure samples are also independent. Two cubic volumes which overlap or share a common planar boundary will not be independent, particularly for the measurement of contiguity which is strongly influenced by the geometry at the boundary. To avoid such a scenario, it is useful to define a separation distance, specific to the particular material, at which microstructural features at two locations are not correlated. For this purpose, we introduce a definition of covariance, which in the X-direction is written as[4,9]

$$
K_{x}(h)=\frac{1}{V} \int I(x, h, y, z) d V
$$




$$
\text { where } I(x, h, y, z)= \begin{cases}1 & \text { if } k(x, y, z)=k(x+h, y, z) \\ 0 & \text { if } k(x, y, z) \neq k(x+h, y, z)\end{cases}
$$

The variable $k$ is used to denote the digitized volume, which contains integer labels for each phase. The variables $x, y$, and $z$ denote spatial coordinates of a given voxel within $k$, and $h$ denotes the offset of the volume $k$ relative to itself. $I$ is a logical variable denoting whether or not a particular voxel in $k$ contains the same phase as the voxel in $k$ spatially offset (in the $\mathrm{x}$ direction, in this case) by the distance $h$. To evaluate Eq. 2, a cubic volume of material, denoted $k$, is extracted from the segmented data set. (For the purposes of determining the covariance, the largest possible cube is taken from the reconstructed data as to include as much material, and therefore produce as accurate a measurement, as possible.) The covariance $K$ of the cube with itself, as a function of the offset distance $h$, gives a measure of the scale over which the microstructural features are correlated. A typical result applying Eq. 2 to a sample in this study is shown in Fig. 1(a).

At small $h$, a structure is highly correlated with itself, and $K$ is close to a value of 1 . As $h$ increases, the correlation decreases and asymptotes to the limit of the sum of the volume fractions of each phase squared, as in Eq. 3.

$$
\lim (h \rightarrow \infty), \quad K_{x}(h)=\sum_{i} \phi_{i}^{2}
$$

The asymptotic limit indicates uncorrelated structures, and can be used to define a correlation length. For each offset step $\mathrm{h}$, the covariance in the $\mathrm{x}, \mathrm{y}$, and $\mathrm{z}$ directions can be averaged to produce an average covariance $\mathrm{K}_{\text {ave }}$. This is only appropriate for isotropic structures in which the curves in the $\mathrm{x}, \mathrm{y}$, and $\mathrm{z}$ directions are similar. For anisotropic structures, the three directions will produce distinctly different curves, and such an operation would not be valid. No 
clear anisotropy was observed for any of the samples in this work. Following the definition used by Laurencin et al., the correlation length is defined as the offset $h^{*}$ at which the covariance is within 5 percent of its asymptotic value,[4]

$$
\frac{K_{a v e}\left(h^{*}\right)-\sum_{i} \phi_{i}^{2}}{\sum_{i} \phi_{i}^{2}} \leq 0.05
$$

It should be noted that the method outlined above to determine the correlation length is, in itself, subject to the RVE volume independence concept. Therefore we arrive at a case of circular logic: the correlation length is being used in a process to help determine the RVE size for a number of other properties, but the correlation length itself cannot be rigorously calculated without knowing the RVE size. While this is true, in practice it has not been a significant issue for two reasons. First of all, the definition of correlation length in Eq. 4 is somewhat arbitrary and should be seen as an approximation. It is meant to give an estimate of the length scale characterizing the structure. Secondly, it is typically clear when not enough material is available for Eq. 2 to produce meaningful results. This has been illustrated in Fig. 1(b). Whereas the covariograms in Fig. 1(a) display the expected behavior and asymptote to a common value, this is not the case in Fig. 1(b). It can be seen that the covariance in the $\mathrm{x}, \mathrm{y}$, and $\mathrm{z}$ directions is not similar, and more significantly they do not seem to asymptote towards the expected limit. This behavior occurs when the cube is small compared to the size of the microstructural features, and indicates not enough of these features have been sampled to provide a meaningful covariogram and correlation length. This has been observed in particular for the samples of processing condition \#5, which were not analyzed any further in this work.

Once the correlation length $h^{*}$ is known, it defines the minimum spatial separation at which two points can be considered independent. Neighboring particles, the centers of which are 
separated by an average distance $h^{*}$, are therefore considered independent, consistent with earlier modeling assumptions. Likewise, neighboring cubic volumes used for analysis must also be separated by this distance $h^{*}$ so their boundaries do not share the same voxels and produce dependent results. The tomography data, consistent with the shape of the FIB-prepared samples, are cylindrical and due to imperfect mounting of the cylinders on the pin during the preparation process are frequently tilted relative to the Cartesian grid, creating oblique cylinders. A simple geometric search routine has been written in MATLAB to determine the number and locations of cubes of a given size $\mathrm{L}^{*}$ that can be extracted from within the constraints of the sample geometry and the required cube separation distance $h^{*}$. Logically, the number of cubes that can be extracted depends on both $\mathrm{L}^{*}$ and $h^{*}$. This procedure can be repeated for different desired $\mathrm{L}^{*}$ using the same tomography data and same $h^{*}$. The results of the cube extraction routine for two different cube sizes are shown in Fig. 2. Note, to test the RVE concept, each cube will only be compared to others of the same $L^{*}$, therefore cubes of different sizes may re-sample the same data.

The largest cube that could be extracted from most samples was 7 microns on a side. The authors acknowledge that a 7 micron cube may not always be large enough to provide representative measurements, and this will be explored in the following sections. Throughout each cylindrical sample, as many cubes as possible were extracted with 7, 6, 5, 4, and 3 micron dimensions. The exact size and orientation of the cylindrical samples dictated how many cubes of each size could be extracted. The number obtained for each size and sample is shown in Table 1. To express the cube dimensions in terms of $\mathrm{L}^{*}$, an average characteristic particle size must be chosen to represent the denominator in Eq. 1. This number is an approximation that must be estimated by the researcher, and for this work was taken as the average correlation 
length $h^{*}$ for all samples created using processing conditions \#1-4, as this represents an average length scale of all features in the samples. The estimate for average particle size was thus taken as 0.77 microns. The cubes measured experimentally in this study therefore correspond approximately to $\mathrm{L}^{*}=3.9,5.2,6.5,7.8$, and 9.1. In future applications of this approach, a researcher could also estimate the characteristic particle size in simpler ways, such as from 2D SEM micrographs, since generally covariograms are not available $a$ priori as presented in this work.

Each cube was analyzed using a set of microstructural characterization codes.[10,11] Properties obtained through the characterization include the volume fraction of each phase, the size distribution of the "particles" of each phase, and the contiguity (or isolation) of that phase's network structure. Some of the codes have been modified slightly from their original formulation, as described below.

The ray tracing method described by Grew et al. and used in this work to determine phase sizes has been modified to provide an improved manner of handling domain boundaries.[11] Following the RVE definition, a cubic volume which we would like to use as representative and descriptive of the total sample could conceptually be replicated and repeated spatially, in a tile-like fashion, to recover the full size of the total sample and with a consistent set of properties and behavior. Therefore, a cube for which we are testing the concept of volume independence can be treated as if it were a single structural unit. Such a representation can be achieved through the use of symmetry boundary conditions at each of the 6 faces of the cube. Rays that are launched from within the structure during the ray shooting routine can either i) intercept another phase of material, thereby terminating the ray propagation, or ii) reach the boundary of the cubic volume. In previous implementations of the code, rays of type (ii) were 
terminated and tabulated along with those of type (i). However, a more realistic implementation within the framework of the RVE concept is to impose symmetry boundaries and have rays "bounce back" into the structure and continue propagating until another phase is intercepted and terminates the ray. Figure 3 shows examples of several rays used to measure the red phase, including one which intercepts the top boundary of the domain and follows the symmetry boundary condition. This methodology will provide a more accurate description of the structure because it prevents the premature termination of rays at the cube boundary, which is not a true physical boundary within the material but rather a boundary created by cropping of the subvolume from a larger domain. After all possible rays have been measured, number-weighted and volume-weighted mean particle sizes are calculated as discussed in the references work.[11]

Contiguity of the real structures is measured using the same region-growing method used for the simulated structures, which has been modified slightly from the version presented by Grew et al. to provide the symmetry and periodic boundary condition cases described previously.

\section{Results: Comparison of Models and Experimental Measurements}

\subsection{General Procedure for Anderson-Darling Goodness-of-Fit Test}

For each $\mathrm{L}^{*}$ and each of four processing conditions, a finite number of independent cubes were analyzed, as described in Table 1. Conceptually however, a nearly infinite number of such cubes could be analyzed if experimental time allowed. From a statistical perspective, this nearly infinite number represents the total population of possible sample cubes which could be examined. In order to have an RVE, it is this total population which we desire to have a standard deviation of properties less than or equal to some acceptable value $\sigma$. But since in reality only a very small sub-set of the population has been analyzed, a statistical test should be performed to 
determine if the sub-set could have come from the total population of cubes with standard deviation $\sigma$ (for the volume fraction model, $\sigma=\sigma_{\phi}$ and for the size distribution model, $\sigma=\sigma_{\text {des }}$ ).

Specifically, the group of cubes for each $\mathrm{L}^{*}$ and each processing condition is tested to see if it could have been taken from a hypothesized normal population of cubes with the same mean property value as that of the group, and standard deviation $\sigma$. Because of the relatively small sample sizes of the experimental data, the Anderson-Darling (A-D) goodness-of-fit test is used which compares the cumulative distribution function of the hypothesized normal distribution with that of the ordered empirical data.[12] The hypothesized normal distribution is defined as having the same mean as the experimental data and standard deviation $\sigma$. The null hypothesis of the test states that the data came from a population with the specified normal distribution, while the alternative hypothesis states the data came from a different distribution. A "p-value" significance level of "p-value" $=0.05$ was used for all cases. (Note, "p-value" as used in this context refers to the significance level of the statistical test as is conventional in the statistical literature, rather than a microstructural volume fraction property. To keep this distinction clear, "p-value" will be placed in quotation when referring to the statistical significance level.) A " $p$ value" of less than 0.05 indicates rejection of the null hypothesis, while a "p-value" greater than 0.05 fails to reject the null hypothesis, indicating the data may have come from the specified normal distribution and therefore displays the requirements for an RVE. The test was performed using the built in Anderson-Darling function in MATLAB (adtest) for each phase of the material, each property (volume fraction and mean particle size), $\mathrm{L}^{*}$ value, and processing condition. (The A-D test was not applied to the experimental contiguity results, as will be discussed in a following section). In certain cases, the data were clearly non-normal due to being close in value to the minimum or maximum of their range, such as volume fractions very nearly equal to zero. 
In these cases, the A-D goodness-of-fit test based on a normal distribution is not valid, and the variation in the data has instead been considered sufficiently small to define an RVE when the total spread is less than $2 \sigma$.

\subsection{Model for Volume Fraction RVE}

Comparison of experimental data with the analytical model for volume fraction is performed in the following manner. First, an acceptable standard deviation of results $\sigma_{\phi}$ is chosen as 0.02 for this work. This means that I seek a cube size for which, if many cubes of that same size were extracted from the total material and analyzed in the same manner, the variation in the measurements of volume fraction would have a standard deviation of 0.02 (or $2 \%$ ).

Next, the experimental results are analyzed using the A-D test to determine at which $\mathrm{L}^{*}$ the required condition of $\sigma_{\phi}=0.02$ is met. The experimental data is analyzed separately for each phase within each set of processing conditions \#1-4. An example of the data is shown in Fig. 4 for the volume fraction of the CFO phase from processing condition \#2. This data set passed the A-D test at $\mathrm{L}^{*}=7.8$. Therefore, any cube larger than this size will be representative for the volume fraction of the $\mathrm{CFO}$ phase. The lower limit for the volume representative cube size for this CFO property is somewhere between $\mathrm{L}^{*}=7.8$ and $\mathrm{L}^{*}=6.5$. Because the experimental sampling has been performed for discrete cube sizes, it is unknown exactly where in this range the true limit occurs, and is therefore conservatively reported as the $\mathrm{L}^{*}$ at which the A-D test is first satisfied, which for this example is $\mathrm{L}^{*}=7.8$.

A comparison of experimental results ( 4 phases and 4 processing conditions for a total of 16 data points) and the analytical model for fixed $\sigma_{\phi}=0.02$ is shown in Fig. 5. The green data points indicate instances in which the A-D test was not valid due to non-normal data, which is 
just at very small volume fractions as expected. The estimated volume fraction $p$, which in a real application of this method is estimated by the researcher, is taken as the average volume fraction of the phase over all 7 micron $\left(\mathrm{L}^{*}=9.1\right)$ cubes measured for that processing condition. (Note, the variable $p$ is used to represent a researcher's estimate of volume fraction which in future implementations of this approach would be approximated a priori, while $\phi$ denotes the soughtafter true volume fraction.) The experimental results show reasonable agreement to the model, with the largest RVE sizes required for compositions with volume fractions close to 0.5 . In addition, in most cases the model is conservative and over-predicts the necessary RVE size, although a number of exceptions are seen at very small volume fractions. This could be due to the strong non-normality of the data near the limit of its range.

For the property of volume fraction, additional comparisons can be made to experimental data in the literature. The results presented in Joos et al. suggest an RVE of a solid oxide fuel cell cathode of about $250 \mu \mathrm{m}^{3}$ (corresponding roughly to $\mathrm{L}^{*}=12$ ) for accurately measuring porosity of $\phi=0.49$ within a standard deviation of about 0.01 (based on Fig. 7 in Joos et al.), which is in reasonable agreement to the model.[5] In addition, Laurencin et al. calculated an RVE size for a solid oxide fuel cell anode of $\mathrm{L}^{*}=14$ for measuring porosity of $\phi=0.5$ also with accuracy of about 0.01 , which is in similar agreement.[4]

\subsection{Model for Particle Size RVE}

The comparison of data to the PSD model is done in a similar fashion. The comparison is performed using a fixed standard deviation (SD) ratio of 10. For definition of the SD ratio and derivation of the analytical model, the reader is referred to part 1 of this manuscript. An estimate for $\sigma_{\mathrm{pop}}$, the standard deviation of particle sizes in the total material domain, must be provided by 
the researcher. In this work, rays obtained during the ray-shooting routine on the 7 micron cubes of the various samples were used. The standard deviation of lengths of all pore, GFCC, CFO, and GDC rays from the 7 micron cubes was measured, providing values of $\sigma_{\text {pop }}=0.31,0.27$, 0.63 , and 1.05 microns, respectively. The SD ratio was then used to calculate $\sigma_{\text {des }}$ which was used in the A-D test to evaluate the experimental data.

Experimental results are shown as data points alongside the analytical model curve in Fig. 6. A green arrow is also shown, indicating a location where 3 data points had very small volume fractions, and large enough cubes were not analyzed to satisfy the RVE criteria. The arrow indicates that the size of the RVEs for those phases must therefore be larger than what was measured.

Reasonable agreement between the data and model is shown in Fig. 6. Some discrepancies seem to appear at the high volume fractions, where a larger RVE is required experimentally than dictated by the model. The authors suggest that the lack of very good agreement may be due to the difficulty in estimating the standard deviation of particle sizes in the total material domain. Methods for improving this approximation represent potential future improvements in the application of the presented approach. However, measurements are still reasonably close to the prediction curve, validating that it provides a reasonable starting point for the prediction of RVE dimensions needed for measuring particle sizes experimentally.

\subsection{Model for Network Contiguity}

The simulation approach presented in Part 1 of this work to describe network contiguity does not provide a closed-form prediction equation as was found for the other two properties. Rather, the variation observed in simulated structures is meant to provide some general guidance 
in the planning of experiments as well as the interpretation of the results for real structures. The contiguity of the GDC-CFO samples in this study was analyzed using the type 1 symmetry/free boundary condition formulation described in Part 1 of this study. Symmetry/free boundaries are chosen in an effort to produce realistic geometric features at the boundaries because they ensure smooth and continuous particle surfaces without creating new, artificial surfaces at the boundaries. Periodic boundary conditions, on the other hand, would generate jagged and discontinuous surfaces when the domain is stacked periodically with itself, and are therefore considered to be a conceptually poor representation of the structure. (Note, in the infinite limit with large cube sizes, the material at the boundaries becomes insignificant in comparison to the internal volume, and the distinction of different boundary conditions should no longer be important). For the four processing conditions and four phases considered, 15 out of the 16 phases contained clusters which were either very nearly completely contiguous or completely isolated for all $\mathrm{L}^{*}$. This was because the compositions of these phases were not near their percolation threshold. However, interesting results were found for the CFO phase of processing condition \#2 because the CFO volume fraction was found to be about $34 \%$, and therefore in the vicinity of its expected percolation threshold. With a phase near the percolation threshold, we would expect to see greater variation in the measured results as well as a dependence on volume size. The contiguity of the phase as a function of $\mathrm{L}^{*}$ is shown in Fig. 7.

Comparison of this result with the simulation results in Fig. 8 (which is equivalent to Fig. 4(a)-(d) in Part 1 of this study) can help provide a description of the structure. At small $\mathrm{L}^{*}$ $\left(L^{*}<6\right)$ erroneous experimental measurements can be observed describing zero contiguity in Fig.

7. Likewise, the simulations in Fig. 8 predict significant scatter of results for $L^{*}=6$, even when 
the phase composition may be well above the percolation threshold. However, in Fig. 7 at larger $\mathrm{L}^{*}$ the experimental errors are not present, and there is a fairly narrow spread of values between 0.9 and 1. Comparing the experimental data points at $\mathrm{L}^{*}=9.1$ (Fig. 7) to the simulation data for $\mathrm{L}^{*}=10$ (yellow symbols, Fig. 8) suggests the phase is likely at least $10 \%$ above its percolation threshold. The percolation threshold predicted by Kuo and Gupta for spherical particles in a 3-D continuous matrix is about $18.7 \%$, meaning a $10 \%$ increase would be about $29 \%$.[13] This value is fairly close to the measured volume fraction of the CFO (34\%) in this sample, indicating the simulations provide a reasonable means of analyzing real data. If the material is thus assumed to be about $10 \%$ above its percolation limit, the simulations for very large $\mathrm{L}^{*}\left(\mathrm{~L}^{*}=100\right)$ provide a predictive capability for the infinite material, and suggest a true contiguity value of about 0.97 .

\section{Conclusions}

Imaging was performed in 3-D using synchrotron-based x-ray nanotomography on composite gas separation membranes synthesized under a variety of conditions. The membranes consisted of three solid phases plus pore space. The microstructures of the samples were evaluated in terms of volume fraction, particle size, and network contiguity. Samples of various sizes were analyzed by splitting large volumes into smaller independent sub-volumes. The variation in characterization results amongst replicate samples of a given sub-volume size was used to evaluate the size at which volume-independent measurements were obtained. The experimental results were compared with predictions from statistical models developed in Part 1 of this work. Reasonable agreement was found between the models, experimental data, and 
several previous reports in the literature, providing confidence in the predictive capabilities of the models as well as their applicability to real samples.

Acknowledgements

Financial support from an Energy Frontier Research Center on Science Based Nano-Structure Design and Synthesis of Heterogeneous Functional Materials for Energy Systems (HeteroFoaM Center) funded by the U.S. Department of Energy, Office of Science, Office of Basic Energy Sciences (Award DE-SC0001061) and the National Science Foundation (Award CBET1134052) are gratefully acknowledged. Samples were provided by Prof. K.S. Brinkman (Clemson University). Portions of this research were carried out at the National Synchrotron Light Source, Brookhaven National Laboratory, with support by the U.S. Department of Energy, Office of Science, Office of Basic Energy Sciences, under contract no. DE- AC02-98CH10886, and at the Stanford Synchrotron Radiation Lightsource, a national user facility operated by Stanford University on behalf of the U.S. Department of Energy, Office of Basic Energy Sciences. Analysis of microstructures was carried out on the HORNET cluster at the University of Connecticut's Booth Engineering Center for Advanced Technology.

\section{References}

[1] Shearing, P. R., Howard, L. E., Jørgensen, P. S., Brandon, N. P., Harris, S. J., 2010, "Characterization of the 3-dimensional microstructure of a graphite negative electrode from a Liion battery," Electrochem. Commun., 12(3), pp. 374-377.

[2] Metcalfe, C., Kesler, O., Rivard, T., Gitzhofer, F., Abatzoglou, N., 2010, "Connected threephase boundary length evaluation in modeled sintered composite solid oxide fuel cell electrodes," J.Electrochem.Soc., 157(9), pp. B1326-B1335. 
[3] Lombardo, J. J., Ristau, R., Harris, W. M., Chiu, W. K. S., 2012, "Focused Ion Beam Preparation of Samples for X-ray Nanotomography," J. Synchrotron Radiat., 19 pp. 789-796.

[4] Laurencin, J., Quey, R., Delette, G., Suhonen, H., Cloetens, P., Bleuet, P., 2012, "Characterisation of Solid Oxide Fuel Cell Ni-8YSZ substrate by synchrotron X-ray nanotomography: from 3D reconstruction to microstructure quantification," J.Power Sources, 198(0), pp. 182-189.

[5] Joos, J., Ender, M., Carraro, T., Weber, A., Ivers-Tiffée, E., 2012, "Representative volume element size for accurate solid oxide fuel cell cathode reconstructions from focused ion beam tomography data," Electrochim.Acta, 82 pp. 268-276.

[6] Rolland du Roscoat, S., Decain, M., Thibault, X., Geindreau, C., Bloch, J. -., 2007, "Estimation of microstructural properties from synchrotron X-ray microtomography and determination of the REV in paper materials," Acta Materialia, 55(8), pp. 2841-2850.

[7] J. Gelb, A. Gu, T. Fong, L. Hunter, S.H. Lau, W. Yun, International Symposium of the Society of Core Analysts, (2011).

[8] Harris, W., Brinkman, K., Lin, Y., Su, D., Cocco, A., Nakajo, A., DeGostin, M., ChenWiegart, Y. K., Wang, J., Chen, F. F., 2014, "Characterization of 3D Interconnected Microstructural Network in Mixed Ionic and Electronic Conducting Ceramic Composites," Nanoscale, 6 pp. 4480-4485.

[9] Kanit, T., Forest, S., Galliet, I., Mounoury, V., Jeulin, D., 2003, "Determination of the size of the representative volume element for random composites: statistical and numerical approach," Int.J.Solids Structures, 40(13-14), pp. 3647-3679.

[10] Grew, K. N., Peracchio, A. A., Joshi, A. S., Izzo Jr., J. R., Chiu, W. K. S., 2010, "Characterization and analysis methods for the examination of the heterogeneous solid oxide fuel cell electrode microstructure. Part 1: Volumetric measurements of the heterogeneous structure," J.Power Sources, 195(24), pp. 7930-7942.

[11] Grew, K. N., Peracchio, A. A., Chiu, W. K. S., 2010, "Characterization and analysis methods for the examination of the heterogeneous solid oxide fuel cell electrode microstructure: Part 2. Quantitative measurement of the microstructure and contributions to transport losses," J.Power Sources, 195(24), pp. 7943-7958.

[12] Anderson, T. W., Darling, D. A., 1954, "A test of goodness of fit," Journal of the American Statistical Association, 49(268), pp. 765-769.

[13] Kuo, C., Gupta, P. K., 1995, "Rigidity and conductivity percolation thresholds in particulate composites," Acta metallurgica et materialia, 43(1), pp. 397-403. 


\begin{tabular}{|l|l|l|l|l|l|l|l|}
\hline $\begin{array}{l}\text { Sample } \\
\text { ID \# }\end{array}$ & $\begin{array}{l}\text { Processing } \\
\text { Condition }\end{array}$ & Location & $\begin{array}{l}7 \mu \mathrm{m} \\
\mathrm{L}^{*}=9.1\end{array}$ & $\begin{array}{l}6 \mu \mathrm{m} \\
\mathrm{L}^{*}=7.8\end{array}$ & $\begin{array}{l}5 \mu \mathrm{m} \\
\mathrm{L}^{*}=6.5\end{array}$ & $\begin{array}{l}4 \mu \mathrm{m} \\
\mathrm{L}^{*}=5.2\end{array}$ & $\begin{array}{l}3 \mu \mathrm{m} \\
\mathrm{L}^{*}=3.9\end{array}$ \\
\hline 1 & 1 & SSRL & 2 & 2 & 3 & 6 & 16 \\
\hline 2 & 4 & SSRL & 0 & 0 & 2 & 3 & 3 \\
\hline 4 & 3 & SSRL & 0 & 0 & 2 & 3 & 6 \\
\hline 5 & 2 & SSRL & 1 & 2 & 4 & 4 & 12 \\
\hline 7 & 1 & NSLS & 1 & 2 & 2 & 6 & 12 \\
\hline 8 & 4 & NSLS & 1 & 1 & 2 & 4 & 12 \\
\hline 10 & 3 & NSLS & 1 & 2 & 2 & 4 & 12 \\
\hline 11 & 2 & NSLS & 2 & 2 & 2 & 6 & 16 \\
\hline 12 & 1 & NSLS & 1 & 2 & 2 & 5 & 12 \\
\hline 13 & 3 & NSLS & 1 & 1 & 2 & 4 & 12 \\
\hline 14 & 2 & NSLS & 2 & 2 & 2 & 6 & 16 \\
\hline 15 & 4 & NSLS & 1 & 2 & 2 & 2 & 6 \\
\hline
\end{tabular}

Table 1: The experimental data used in this study, including the processing conditions, where imaging was performed, and the number of cubes of each size that were extracted from each sample. 

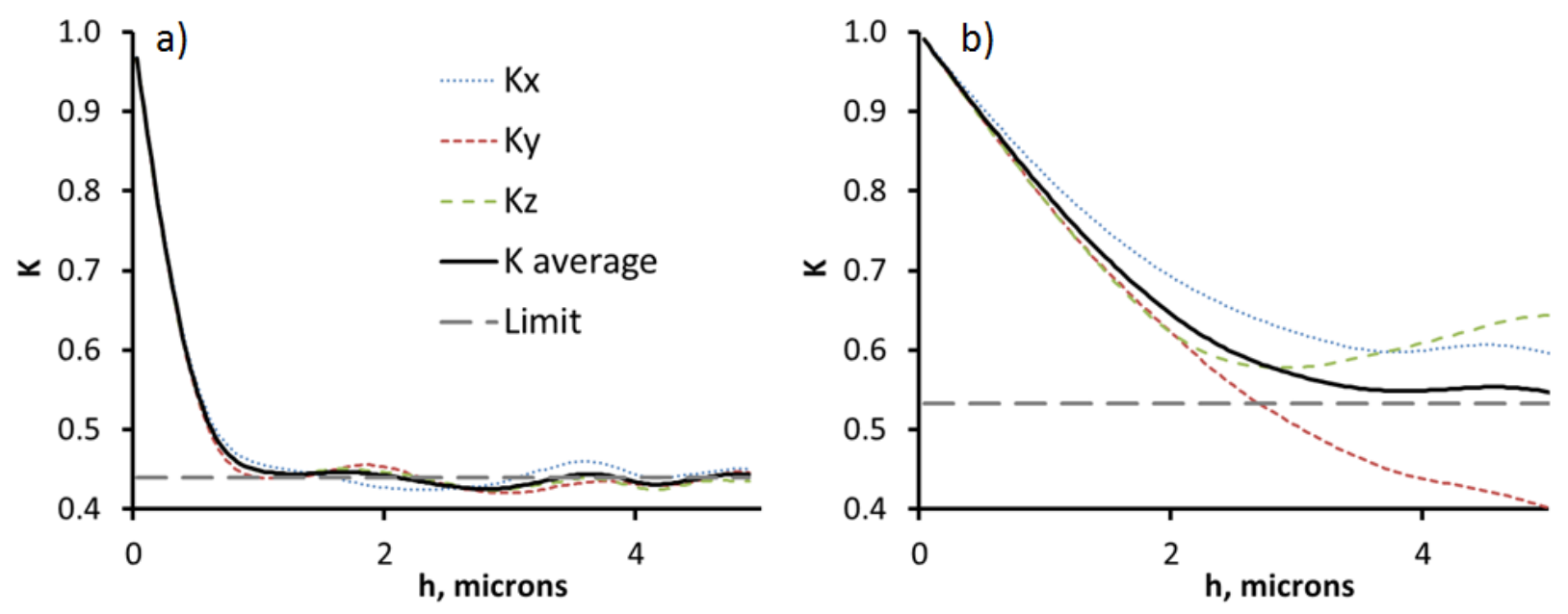

Figure 1: Covariograms for two different samples. In (a), a sufficiently large volume of material is sampled to produce a covariogram which display the expected asymptotic limiting behavior. In (b), very large features relative to the volume size prevented the measurement of a meaningful covariogram. 

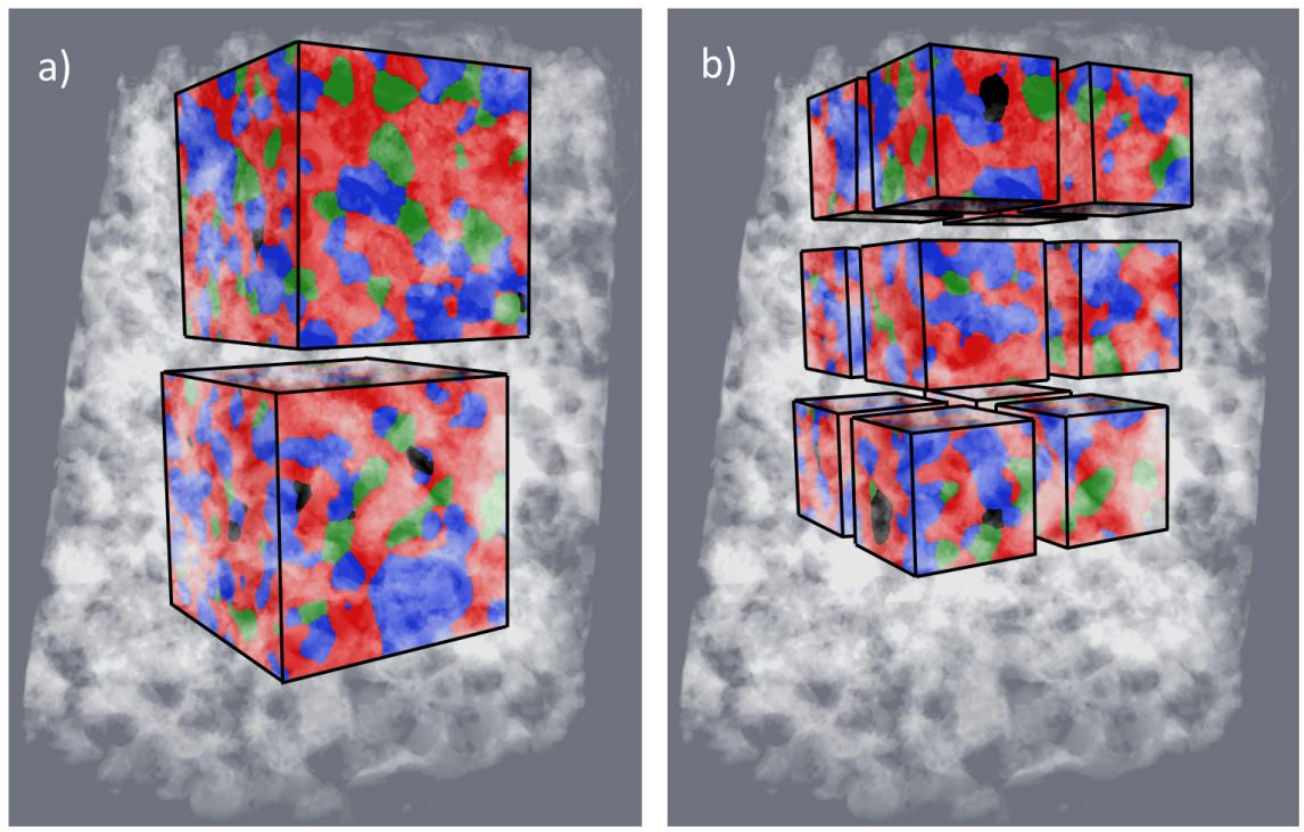

Figure 2: Cubic sub-volumes are extracted from within the cylindrical samples analyzed using $\mathrm{x}$ ray nanotomography. Cubes of different sizes are used, while maintaining a separation between cubes equal to the sample's covariance length $h^{*}$. Cubes of edge length 6 microns (a) and 3 microns (b) are shown. 


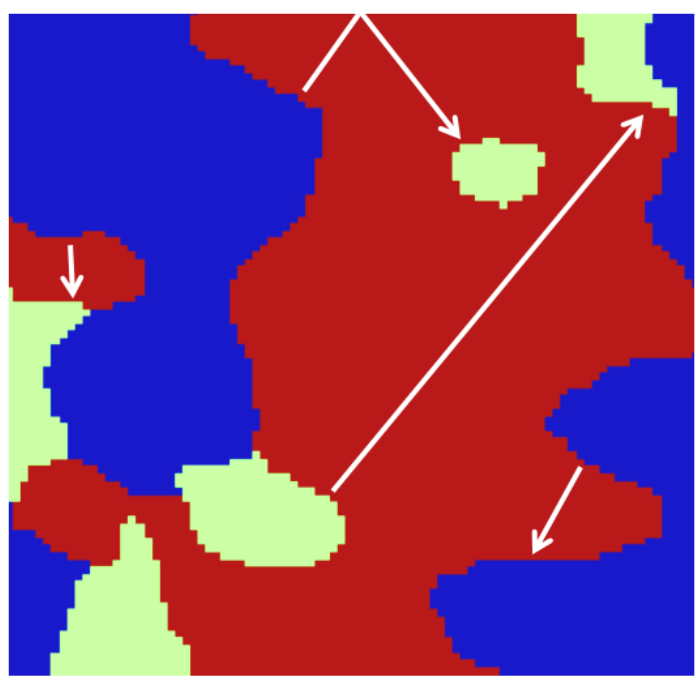

Figure 3: Depiction of the ray shooting method to measure the size distribution of the red phase. Implementation of symmetry boundary conditions is demonstrated with the ray on the top boundary of the domain. 


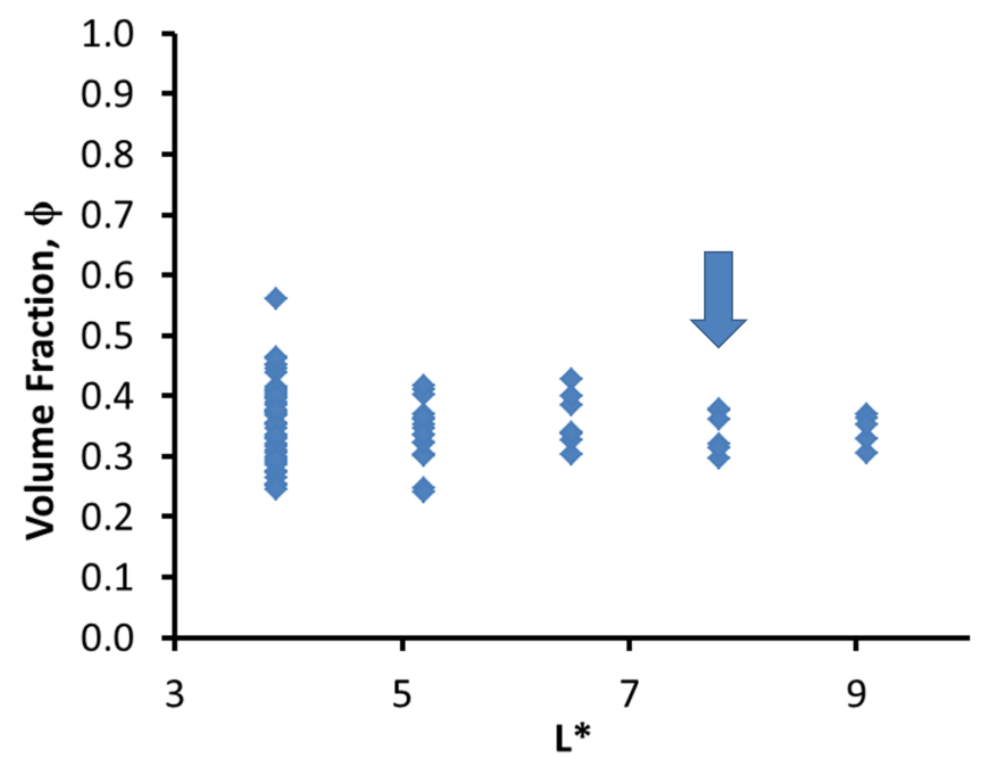

Figure 4: Experimental results for the volume fraction of the CFO phase in the sample produced using processing condition \#2. The blue arrow indicates the value of $\mathrm{L}^{*}$ at which the data passes the Anderson-Darling test with $\sigma_{\phi}=0.02$. 


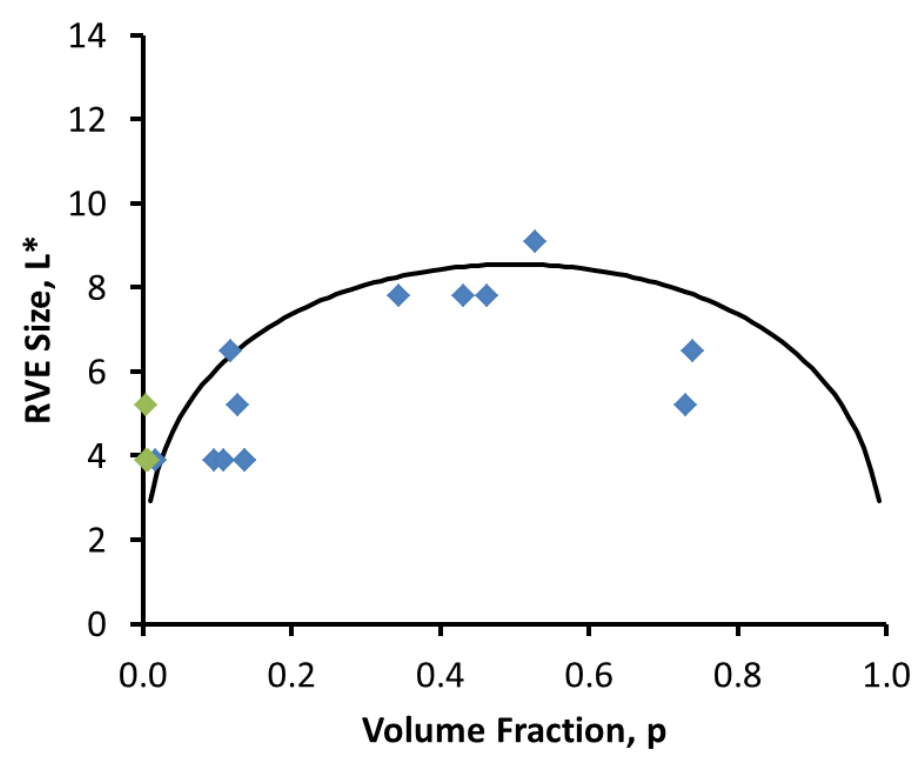

Figure 5: Comparison of the volume fraction analytical model with experimental results for fixed $\sigma_{\phi}=0.02 . p$ represents the researcher's estimate of volume fraction. The model is shown by the black curve, while the points represent experimental results. The green points are instances where strongly non-normal data prevented the use of the Anderson-Darling test, as discussed in the text. 


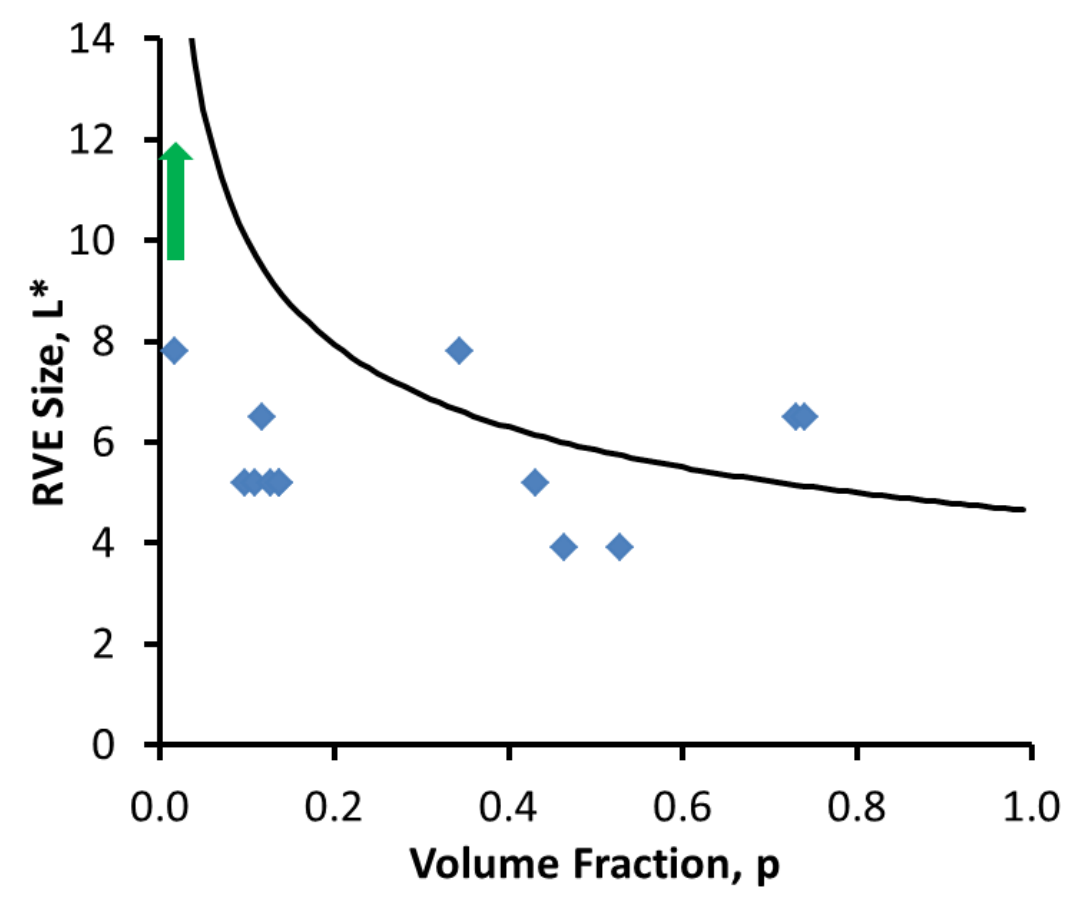

Figure 6: Comparison of the size distribution RVE model with experimental results for a fixed standard deviation ratio of 10. $p$ represents the researcher's estimate of volume fraction. The model is shown by the black curve, while the points represent experimental results. The green arrow is meant to depict several instances where an RVE size was not determined (and must therefore be larger than the maximum size which was measured). 


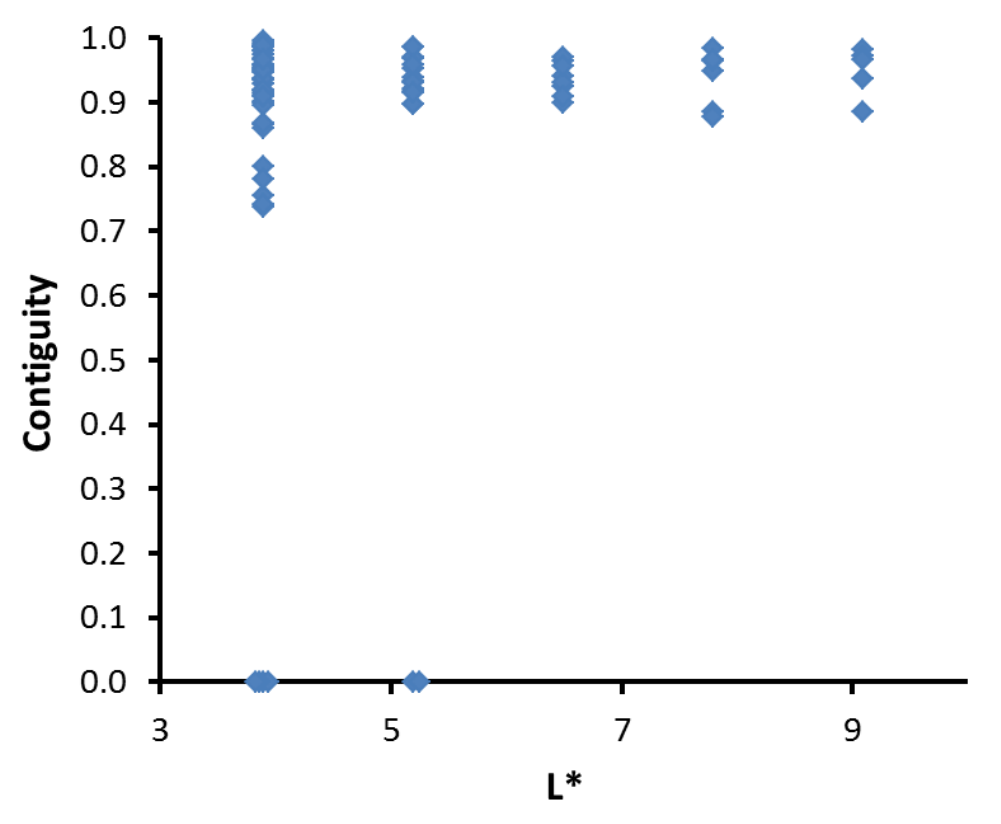

Figure 7: Experimental results for the contiguity of the CFO phase of the sample produced with processing condition \#2. Symmetry/free boundary conditions were used to measure contiguity. For small L*, several measurements were obtained with zero contiguity because these small sizes constitute non-representative volumes and can, by chance, contain only limited structures which do not span the volume. Note these non-representative measurements are not obtained for larger $\mathrm{L}^{*}$. 


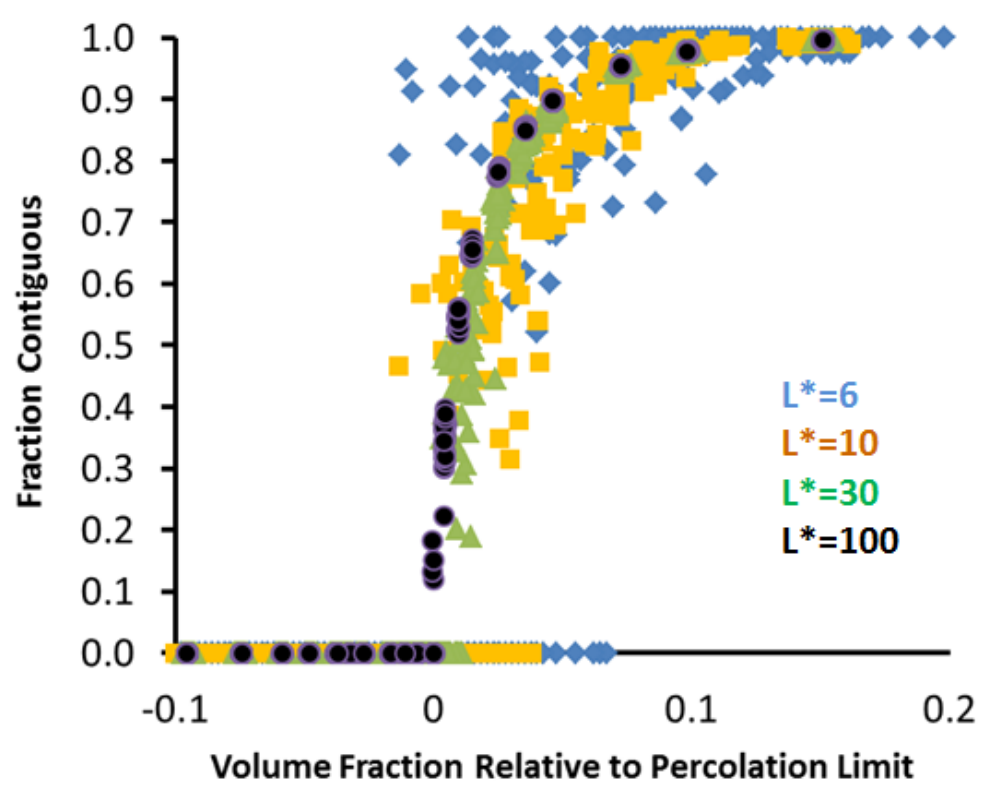

Figure 8: Simulation results of network contiguity based on site percolation on a 3-D simple cubic lattice. 